\title{
Ankle ultrasound tenosynovitis is a significant predictor of DAS28 - defined rheumatoid arthritis activity
}

\author{
Luminita Enache ${ }^{1,2}$, Claudiu C. Popescu ${ }^{1,2}$, Catalin Codreanu ${ }^{1,2}$, Maria Suta ${ }^{1}$ \\ ${ }^{1}$ Dr. Ion Stoia Clinical Centre of Rheumatic Diseases, Bucharest, Romania \\ 2"Carol Davila" University of Medicine and Pharmacy, Bucharest, Romania \\ ${ }^{3}$ Faculty of Medicine, Ovidius University of Constanta, Constanta, Romania
}

\begin{abstract}
Objective. The study aimed to observe the frequency of ultrasound-defined tenosynovitis in ankle tendon and to evaluate the relationship of ankle tenosynovitis with clinical examination and rheumatoid arthritis (RA) activity measures.

Methods. RA patients were recruited in 2018 in the random order of presentation from the out-patient clinic. On the same day of inclusion, all patients underwent clinical examination, laboratory tests (inflammatory markers), ankle ultrasound and patient-reported outcomes.

Results. the study included 183 patients with established RA, mostly women (86.3\%), with mean age of 57.3 years. The most frequent tenosynovitis was observed in the tibialis posterior tendon (TP; $40.4 \%$ ), followed by the peroneus longus $(23.0 \%)$ and peroneus brevis $(18.0 \%)$ tendons. Compared to patients without TP tenosynovitis, patients with TP tenosynovitis had significantly higher titres of rheumatoid factors (RF; median of $123 \mathrm{IU} / \mathrm{mL} \mathrm{com}-$ pared to $64 \mathrm{IU} / \mathrm{mL} ; p=0.023)$. Clinically tender $(55.2 \%)$ and swollen $(30.6 \%)$ ankles were 4.2 and respectively 11.6 times more likely to reveal tenosynovitis on ultrasound. The presence of ankle tenosynovitis was associated with higher disease activity measures. DAS28 increased proportionally and significantly with the number of ankles with tenosynovitis, the grade of ankle tenosynovitis and power Doppler activity. The absence of ankle tenosynovitis independently and significantly decreased DAS28 with 1.2 points $(p<0.001)$.

Conclusions. the most frequent ankle tenosynovitis observed in RA patients involves the TP tendon, which is associated with higher titres of RF. Swollen ankles are more specific and better predictors of ultrasound-defined ankle tenosynovitis, which has a directly proportional relationship with disease activity in RA. Disease activity scores should include clinical evaluation of ankles and ultrasound information.
\end{abstract}

Keywords: rheumatoid arthritis, ankle, ultrasound, tenosynovitis

\section{INTRODUCTION}

It is not uncommon in clinical practice for rheumatoid arthritis (RA) to start with ankle involvement or to exhibit persistent ankle involvement despite achieving disease remission defined by composite scores (e.g. DAS28 [1]). In fact, in a recent large national cohort study, $43.8 \%$ of RA patients reported that their disease started with ankle or foot symptoms [2]. Rheumatologists can attest the existence of patients with chronic clinical ankle arthritis despite the most modern of treatments. Acknowledging this observation, the European League against Rheumatism (EULAR) taskforce recommended to include ankles in the Boolean definition of RA remission [3], underlying the concept that true remission should be reflected in all synovial joints. The task of clinically evaluating ankle joints can prove to be difficult because of their complex anatomy and multiple possible confounders which can decrease the accuracy of detection of RA-related involvement (for example obesity, chronic venous insufficiency etc.). Musculoskeletal ultrasound can increase the objectivity of this evaluation, with the advantage of discriminating between synovial inflammation in joint and tendon sheath ankle involvement [4]. Although the clinically-justified recommendation to evaluate ankles in RA management is present in many practical guidelines, it is supported by few and low quality literature evidence [5]. The rarity of ankle studies in RA is certain, but a more important problem is that some 
of this evidence is contradictory. Using both clinical examination and ultrasound, Elsaman et al. [6] observed that the most frequent ankle ultrasound finding was tenosynovitis $(30,2 \%)$, which was also the earliest pathologic finding. The authors also reported that the most common tendon affected by tenosynovitis was the tibialis anterior (TA; 22.2\%), followed by the tibialis posterior (TP; 20.6\%). However, Gutierrez et al. [7] agreed that the most common ultrasound anomaly was tenosynovitis, but they reported that the most frequent tendons involved were $\mathrm{TP}$ (31.9\%), peroneus longus (PL; 26.9\%) and peroneus brevis (PB; 23.6\%), with no significant association of ankle ultrasound findings with DAS28. Investigating clinical associations of ankle tenosynovitis, Suzuki et al. observed a positive association between ankle tenosynovitis and rheumatoid factor (RF) levels [8] and the fact that tenosynovitis was significantly more common in early RA ankles (disease duration under 6 months) [9]. Ankle tenosynovitis is responsible for secondary tendon dysfunction [10] and it is responsive to RA treatment [11].

In a recent study [12], we aimed to investigate the relationship between ankle involvement and disease activity in RA, from a clinical and ultrasound perspective, limiting the analysis to ankle synovial hypertrophy (SH) detection and relevance. We observed that both clinically tender and swollen ankles were approximately 3 times more likely to reveal ultrasound ankle joint SH. Furthermore, the presence, grade and power Doppler (PD) activity of ankle SH were predictive of significantly higher disease activity measures. We concluded that clinical ankle involvement and ultrasound-detected ankle SH have a directly proportional relationship with disease activity in RA. Using the same data set in the current study, we aimed to observe if these conclusions were also true for ankle tenosynovitis in RA. More specifically, the study aimed to observe the frequency of ultrasound-defined tenosynovitis in ankle tendon and to evaluate the relationship of ankle tenosynovitis with clinical examination and disease activity measures.

\section{METHODS}

\section{Patient selection}

RA patients were recruited in 2018 in the random order of presentation to the out-patient clinic from an academic hospital (Clinical Centre for Rheumatic Diseases, Bucharest, Romania). Study inclusion re- quired fulfilment of the 2010 American College of Rheumatology (ACR)/EULAR classification criteria of RA [13] and age above 18 years. Exclusion criteria consisted of history or current ankle deformity/surgery, local complex regional pain syndrome, fibromyalgia, injectable glucocorticoids (pulse-therapy, intramuscular, intra-articular injections) in the month prior to study inclusion. Oral glucocorticoids ( $\leq 10 \mathrm{mg}$ prednisone equivalent) which were stable in the month prior to study inclusion were allowed. Similarly, stable doses of nonsteroidal anti-inflammatory drugs (NSAIDs) in the last week were allowed. The study was approved by the local ethics committee and all patients gave written informed consent prior to enrolment and any study procedures. On the same day of study inclusion, all patients underwent clinical examination, laboratory tests, ankle ultrasound and completed independently patient-reported outcomes: patient global assessment of general health (PtGA; on a $100 \mathrm{~mm}$ visual analogue scale) and health assessment questionnaire (HAQ).

\section{Clinical and laboratory evaluation}

All clinical examinations were done by the same senior rheumatologist, blinded to laboratory and ultrasound results. The 28 joints included in DAS28 and both ankles were examined by inspection, palpation and active movement at each compartment, screening for signs of inflammation (pain and/or swelling). Concomitant local ankle pathology was also noted: hallux valgus, pes planus, chronic venous insufficiency. A clinical interview noted aspects of general medical history, RA history and current anti-rheumatic treatment. Obesity was defined as body mass index above $30 \mathrm{~kg} / \mathrm{m}^{2}$. Standard 28 -joint tender/swollen counts were performed and disease activity scores (DAS) were computed: DAS28 [1] using C-reactive protein (CRP, which is better correlated with synovial inflammation than erythrocyte sedimentation rate - ESR [14]) and SDAI (simplified disease activity index) [15]. Laboratory tests included CRP (normal $<5 \mathrm{mg} / \mathrm{dl}$ ), ESR (normal $<20$ $\mathrm{mm} / \mathrm{h}$ ), rheumatoid factor $(\mathrm{RF}$; normal $<30 \mathrm{IU} / \mathrm{mL}$ ) and anti-citrullinated protein antibodies (ACPA; normal $<20 \mathrm{IU} / \mathrm{ml})$.

\section{Ankle ultrasound}

Ankle ultrasound scans were performed and interpreted by a single rheumatologist with more than 7 years of experience in RA patients (Figure 1), on 
the same day as the clinical examination and blinded to its result. Using an Esaote MyLabTwice machine (12-18 MHz linear transducer), both ankles were scanned according to the EULAR-recommended ankle technique [16]. First, ankles were scanned in grey scale, followed by power Doppler (PD) tech- nique, using constant settings (gain just below the noise level, $750 \mathrm{~Hz}$ pulse repetition frequency, 8-10 MHz Doppler frequency, low wall filter). The following tendons were examined: tibialis anterior (TA), extensor hallucis longus (EHL), extensor digitorum longus (EDL), tibialis posterior (TP), flexor

TABLE 1. General characteristics of RA patients $(n=183)$

\begin{tabular}{rlrl} 
women & $158(86.3 \%)$ & RF (IU/mL) & $75.3(0-1600)$ \\
age (y) & $57.3(12.5)$ & RF positive & $118(64.5 \%)$ \\
disease duration (y) & $11.2(10.3)$ & ACPA (IU/mL) & $166.1(0-530)$ \\
NSAIDs & $48(26.2 \%)$ & ACPA positive & $139(76.0 \%)$ \\
glucocorticoids & $32(17.5 \%)$ & TJC28 & $4(0-25)$ \\
csDMARDs & $155(84.7 \%)$ & $\geq 1$ tender ankle & $101(55.2 \%)$ \\
methotrexate & $88(48.1 \%)$ & SJC228 & $1(0-24)$ \\
$>1$ csDMARD & $17(9.3 \%)$ & 1 swollen ankle & $56(30.61026+\%)$ \\
bDMARDs & $64(35.0 \%)$ & ESR (mm/h) & $34(2-98)$ \\
bDMARD monotherapy & $1(0.5 \%)$ & CRP $(\mathrm{mg} / \mathrm{L})$ & $9.2(0.2-196)$ \\
T2DM & $17(9.3 \%)$ & PtGA (mm) & $42.6(25.9)$ \\
hallux valgus & $58(31.7 \%)$ & PhGA (mm) & $31.5(22.8)$ \\
CVI & $38(20.8 \%)$ & DAS28 2 ESR & $4.4(1.7)$ \\
rheumatoid foot & $18(9.8 \%)$ & DAS28CRP & $3.8(1.7)$ \\
obesity & $4(2.2 \%)$ & SDAI & $18.3(15.7)$ \\
pes planus & $4(2.2 \%)$ & HAQ & $1.5(0.8)$ \\
fibromyalgia & $1(0.5 \%)$ & & \\
\hline
\end{tabular}

notes: normally-distributed continuous variables are reported as "mean (standard deviation)"; non-normally distributed continuous variables are reported as "median (minimum-maximum)"; nominal variables are reported as "count (percentage from sample)"; ACPA - anti-citrullinated protein antibodies; b/cs/tDMARDs - biologic/conventional synthetic/targeted diseasemodifying anti-rheumatic drugs; CRP - C-reactive protein; CVI - chronic venous insufficiency; DAS - disease activity score; ESR - erythrocyte sedimentation rate; HAQ - health assessment questionnaire; IU - international units; NSAIDs - non-steroidal anti-inflammatory drugs; $\mathrm{Ph} / \mathrm{tGA}$ - physician/patient global assessment of disease activity; RF - rheumatoid factor; SDAI - simplified disease activity index; S/TJC - swollen/tender joint count; T2DM - type 2 diabetes mellitus; y - years.

\section{TABLE 2. Regression analysis}

\section{1. binary logistic regression models ${ }^{*}$ to predict ankle tenosynovitis}

\begin{tabular}{rcccr} 
& Nagelkerke $\mathrm{R}^{2}$ & $\chi^{2}(13)^{\#}$ & OR $(95 \% \mathrm{CI})$ & $\mathrm{p}$ \\
\cline { 2 - 5 } a) $\geq 1$ tender ankle joint & 0.465 & 77.6 & $4.18(1.83 ; 9.54)$ & 0.001 \\
b) $\geq 1$ swollen ankle joint & 0.497 & 84.4 & $11.63(3.39 ; 40.0)$ & $<0.001$
\end{tabular}

2. multiple linear regression models ${ }^{*}$ to predict DAS28 ${ }_{C R P}$

\begin{tabular}{rcccr} 
& adjusted R $^{2}$ & $\mathrm{~F}(4,180)^{\#}$ & $\mathrm{~B}(95 \% \mathrm{CI})$ & $\mathrm{p}$ \\
\cline { 2 - 5 } a) TS presence $=0$ & 0.487 & 50.3 & $-1.18(-1.54 ;-0.82)$ & $<0.001$ \\
b) TS grade $=0$ & 0.462 & 45.0 & $-1.02(-1.42 ;-0.63)$ & $<0.001$ \\
c) TS PD presence $=0$ & 0.522 & 68.4 & $-1.32(-1.68 ;-0.96)$ & $<0.001$ \\
\hline
\end{tabular}

* all models included: gender, age (years), disease duration (years), CRP (mg/L; only in models 1.a and 1.b, significant in models 1.a and 1.b), PtGA (mm; significant in model 1.a), HAQ (significant in models 2.a, 2.b and 2.c), seropositivity (RF or/and ACPA positive; significant in model 1.b), csDMARDs, bDMARDs (significant in models 2.a, 2.b and 2.c), glucocorticoids, NSAIDs, ankle confounders (T2DM, hallux valgus, CVI, rheumatoid foot, obesity, pes planus, fibromyalgia; significant in models $1 . a$ and 1.b) and one clinical/ ultrasound ankle variable. $\# p<0.001$. ACPA anti-citrullinated protein antibodies; b/csDMARDs - biologic/conventional synthetic diseasemodifying anti-rheumatic drugs; CI - confidence interval; CRP - C-reactive protein; CVI - chronic venous insufficiency; DAS - disease activity score; HAQ - health assessment questionnaire; NSAIDs - non-steroidal anti-inflammatory drugs; OR - odds ratio; PD - power Doppler; PtGA - patient global assessment; RF - rheumatoid factor; SH - synovial hypertrophy; T2DM - type 2 diabetes mellitus. 
TABLE 3. Disease activity indices and patient-reported outcomes according to ankle tenosynovitis presence $(n=183)$

\begin{tabular}{|c|c|c|c|c|c|c|}
\hline & \multicolumn{2}{|c|}{ TA TS $(7.7 \%)$} & \multicolumn{2}{|c|}{ EHL TS $(3.8 \%)$} & \multicolumn{2}{|c|}{ EDL TS (14.8\%) } \\
\hline & no $(n=169)$ & yes $(n=14)$ & no $(n=176)$ & yes $(n=7)$ & no $(n=156)$ & yes $(n=27)$ \\
\hline TJC28 & 4 & $8^{\#}$ & 4 & $8^{\&}$ & 3 & $6 \&$ \\
\hline SJC28 & 1 & $11^{*}$ & 1 & $13^{\#}$ & 1 & $7^{*}$ \\
\hline $\operatorname{ESR}(\mathrm{mm} / \mathrm{h})$ & 32 & 47 & 34 & 68 & 32 & $52^{\#}$ \\
\hline CRP (mg/l) & 8 & $34^{\&}$ & 8 & $38^{\&}$ & 8 & $34^{\#}$ \\
\hline PtGA (mm) & 40 & $55^{\#}$ & 40 & $90^{\#}$ & 40 & 50 \\
\hline PhGA (mm) & 28 & $50^{*}$ & 30 & $70^{\#}$ & 29 & $45^{\#}$ \\
\hline DAS28 & 4.5 & $5.9^{*}$ & 4.5 & $6.8^{\#}$ & 4.4 & $5.7^{\#}$ \\
\hline DAS28 & 3.8 & $5.3^{*}$ & 3.8 & $6.2^{\#}$ & 3.8 & $5.1^{\#}$ \\
\hline SDAI & 14 & $33^{*}$ & 14 & $45^{\#}$ & 14 & $31^{*}$ \\
\hline \multirow[t]{3}{*}{ HAQ } & 1.5 & $2.1^{\#}$ & 1.5 & $2.6^{\#}$ & 1.5 & 1.9 \\
\hline & \multicolumn{2}{|c|}{ PL TS $(23.0 \%)$} & \multicolumn{2}{|c|}{ PB TS $(18.0 \%)$} & \multicolumn{2}{|c|}{ TP TS $(40.4 \%)$} \\
\hline & no $(n=141)$ & yes $(n=42)$ & no $(n=150)$ & yes $(n=33)$ & no $(n=109)$ & yes $(n=74)$ \\
\hline TJC28 & 3 & $8^{*}$ & 3 & $7^{*}$ & 2 & $7^{*}$ \\
\hline SJC28 & 1 & $5^{*}$ & 1 & $6^{*}$ & 0 & $5^{*}$ \\
\hline $\operatorname{ESR}(\mathrm{mm} / \mathrm{h})$ & 31 & $45^{*}$ & 32 & $50^{*}$ & 28 & $44^{*}$ \\
\hline $\mathrm{CRP}(\mathrm{mg} / \mathrm{l})$ & 7 & $19^{*}$ & 7 & $18^{*}$ & 6 & $18^{*}$ \\
\hline PtGA (mm) & 40 & $60^{*}$ & 40 & $60^{*}$ & 30 & $55^{*}$ \\
\hline $\operatorname{PhGA}(\mathrm{mm})$ & 20 & $46^{*}$ & 21 & $41^{*}$ & 20 & $40^{*}$ \\
\hline DAS28 $8_{\text {ESR }}$ & 4.0 & $5.7^{*}$ & 4.2 & $5.8^{*}$ & 3.6 & $5.7^{*}$ \\
\hline $\mathrm{DAS} 28_{\mathrm{CRP}}$ & 3.4 & $4.9^{*}$ & 3.6 & $5.4^{*}$ & 2.9 & $5.0^{*}$ \\
\hline SDAI & 11 & $26^{*}$ & 13 & $34^{*}$ & 9 & $26^{*}$ \\
\hline \multirow[t]{3}{*}{ HAQ } & 1.4 & $2.1^{*}$ & 1.5 & $2.1^{*}$ & 1.4 & $1.9^{\&}$ \\
\hline & \multicolumn{2}{|c|}{ FDL TS $(9.8 \%)$} & \multicolumn{2}{|c|}{ FHL TS $(10.4 \%)$} & \multicolumn{2}{|c|}{ any ankle TS (52.5\%) } \\
\hline & no $(n=165)$ & yes $(n=18)$ & no $(n=164)$ & yes $(n=19)$ & no $(n=87)$ & yes $(n=96)$ \\
\hline TJC28 & 4 & $8^{\#}$ & 4 & $8 \#$ & 2 & $7 *$ \\
\hline SJC28 & 1 & $6^{*}$ & 1 & $5^{\#}$ & 0 & $5 *$ \\
\hline $\operatorname{ESR}(\mathrm{mm} / \mathrm{h})$ & 34 & 38 & 32 & 44 & 26 & $44^{*}$ \\
\hline $\mathrm{CRP}(\mathrm{mg} / \mathrm{l})$ & 8 & $18^{\#}$ & 8 & 13 & 3 & $18 *$ \\
\hline $\operatorname{PtGA}(\mathrm{mm})$ & 40 & $55^{\&}$ & 40 & $70^{\#}$ & 30 & $57 *$ \\
\hline PhGA (mm) & 29 & $40^{\&}$ & 26 & $40^{\#}$ & 17 & $40^{*}$ \\
\hline DAS2 $28_{\text {ESR }}$ & 4.4 & $5.7^{\#}$ & 4.4 & $5.8^{\#}$ & 3.4 & $5.6^{*}$ \\
\hline DAS2 $8_{\mathrm{CRP}}$ & 3.8 & $5.1^{*}$ & 3.8 & $4.7^{\&}$ & 2.6 & $4.9^{*}$ \\
\hline SDAI & 14 & $26^{*}$ & 14 & $26^{\#}$ & 7 & $24^{*}$ \\
\hline HAQ & 1.5 & 1.9 & 15 & 2.0 & 1.3 & $1.9 *$ \\
\hline
\end{tabular}

Notes: TS for each tendon is counted if present on either right or left or both ankles; values represent medians which were compared with Mann Whitney tests with the following levels of significance: * $\mathrm{p}$ $\leq 0.001 ; \# \mathrm{p} \leq 0.01 ; \& \mathrm{p}<0.05$; unmarked $\mathrm{p}>0.05$; CRP - C-reactive protein; DAS - disease activity score; EDL - extensor digitorum longus; EHL - extensor hallucis longus; ESR - erythrocyte sedimentation rate; FDL - flexor digitorum longus; FHL - flexor hallucis longus; HAQ - health assessment questionnaire; PB - peroneus brevis; PL - peroneus longus; Ph/tGA - physician/patient global assessment of disease activity; SDAI - simplified disease activity index; S/TJC - swollen/tender joint count; TA - tibialis anterior; TP - tibialis posterior; TS - tenosynovitis.

digitorum longus (FDL), flexor hallucis longus (FHL), peroneus longus (PL) and brevis (PB). Tenosynovitis was defined and quantified using OMERACT recommendations [16].

\section{Statistics}

Distribution normality was assessed using descriptive statistics, normality and stem-and-leaf plots, and Kolmogorov-Smirnov tests. Differences of continuous variables (e.g. DAS28) among categorical variables (e.g. PD presence or tenosynovitis grades) were tested with non-parametric tests for independent variables (Mann-Whitney and Kruskal-
Wallis tests respectively). Post-hoc Bonferoni pairwise comparisons of Kruskal-Wallis test results were done to identify significant differences among categories of multi-level nominal variables (e.g. number of tendons with tenosynovitis). The ability of clinical findings (tender/swollen ankle) to predict ultrasound-defined ankle tenosynovitis was studied using specificity, sensitivity and binary logistic regression and they were compared using the McNemar's test. DAS2 $8_{\text {CRP }}$ prediction was studied with standard multiple linear regression models built using SPSS automatic linear modelling with forward stepwise selection method, information criterion for entry/removal 
and $95 \%$ confidence interval. The statistical tests were considered significant if $p<0.05$ and were computed with IBM SPSS Statistics version 25.0 for Windows (Armonk, NY, IBM Corp.).

\section{RESULTS}

The study included 183 patients with established RA (Table 1), mostly women $(86.3 \%)$, with a mean age of 57.3 (12.5) years. The most frequent tenosynovitis was observed in the TP tendon $(40.4 \%)$, followed by the PL (23.0\%) and PB (18.0\%;) tendons (Table 3). Compared to patients without TP tenosynovitis (59.6\%), patients with TP tenosynovitis (40.4\%) had significantly lower disease duration (median of 7.0 compared to 8.5 years; $\mathrm{p}=0.043$ ), higher DAS28 ${ }_{\text {CRP }}$ (median of 5.0 compared to $2.8 ; \mathrm{p}$ $<0.001$ ), higher HAQ score (median of 1.9 compared to $1.5 ; \mathrm{p}=0.037$ ) and higher titres of RF (median of $123 \mathrm{IU} / \mathrm{ml}$ compared to $64 \mathrm{IU} / \mathrm{ml} ; \mathrm{p}=0.023$ ), with similar titres of ACPA ( $p>0.05)$.

Clinical examination revealed 101 (55.2\%) patients with at least one tender ankle, of whom 70 had tenosynovitis in at least one ankle, producing $60.6 \%$ sensitivity and $64.4 \%$ specificity for clinical examination to detect ultrasound-defined tenosynovitis. Similarly, 56 (30.6\%) patients had at least one swollen ankle at clinical examination, of whom 48 had ankle tenosynovitis on ultrasound (50.0\% sensitivity and $90.8 \%$ specificity). Regression analysis revealed that both clinically tender and swollen ankles were 4.2 and respectively 11.6 times more likely to reveal tenosynovitis on ultrasound (Table 2). The difference between the ability of clinically tender and swollen ankles to predict tenosynovitis presence on ultrasound was significant (McNemar's $\mathrm{p}=0.034$ ) in favour of swollen ankles.

The presence of ankle tenosynovitis was associated with higher disease activity measures (Table 3 ). For example, compared to patients without tenosynovitis in any ankle tendon $(87 ; 47.5 \%)$, those with at least one tenosynovitis $(96 ; 52.4 \%)$ had significantly higher median DAS28 ${ }_{\text {CRP }}$ (4.9 compared to 2.6; $\mathrm{p}<$ 0.001 ) and HAQ scores (1.9 compared to $1.3 ; \mathrm{p}<$ $0.001)$. DAS $28_{\text {CRP }}$ values increased proportionally and significantly with the number of ankles with tenosynovitis (Figure 1A): a patient with one tendon with tenosynovitis had a significantly higher median DAS28 $8_{\text {CRP }}$ than a patient with no tenosynovitis (4.67 compared to 2.64; $\mathrm{p}=0.003$ ). The grade of ankle tenosynovitis behaved similarly: Figure 1B illus- trates how patients with grade 1 ankle tenosynovitis had significantly higher median DAS28 ${ }_{\text {CRP }}$ compared to patients with grade 0 (4.79 compared to $2.61 ; \mathrm{p}=0.001)$. PD activity of ankle tenosynovitis produced similar results (Figure 1C): PD signal presence was associated with higher median DAS$28_{\text {CRP }}(\mathrm{p}=0.001)$. The presence of ankle tenosynovitis and its grades and PD presence in ankle tenosynovitis produced identical significant differences regarding all other disease activity measures reported in Table 3 ( $p<0.03$; data not shown). Ankle tenosynovitis had an independent effect on DAS$28_{\mathrm{CRP}}$-defined disease activity: for example, taking into account all other significant covariates in the regression model (bDMARD, HAQ), the absence of ankle tenosynovitis independently and significantly decreased DAS2 $8_{\text {CRP }}$ with 1.2 points $(\mathrm{p}<0.001$; Table 2).

\section{DISCUSSIONS}

Our data revealed that the most frequent ankle site for ultrasound-defined tenosynovitis was TP (40.4\%). TP was also the leading site for tenosynovitis detection reported by Gutierrez et al. [7] (31.9\%) and the second one in the study of Elsaman et al. [6] (20.6\%). The significantly higher frequency of TP tenosynovitis in our study sample can be interpreted in the context inter-observer variability, technical difference of ultrasound machines and different RA phenotypes included in the study. The TP tendon has an important effect of stabilizing the lower leg through its functional role in inversion, plantar flexion and supporting the arch of the foot. This explains why RA involvement of its tendon can result in a characteristic plano-valgus deformity of the foot $[10,17,18]$. Some authors have argued for the use of isolated talonavicular arthrodesis in patients with TP tendon dysfunction in order to prevent further anatomical deformity of the ankle [19]. If the aim of RA treatment is to preserve joint function, the screening of TP tenosynovitis should represent an important step in assessing walking and gait in RA patients. The association of TP tenosynovitis with higher RF titres (also noted by Suzuki et al. [8]), but not with ACPA is puzzling. High RF titres are specific for RA and their presence is considered to be a prognostic factor since seropositivity is associated with disease progression [20] and mortality [21]. The link between this possibly confounding association may be explained by the levels of pro-inflammatory cy- 

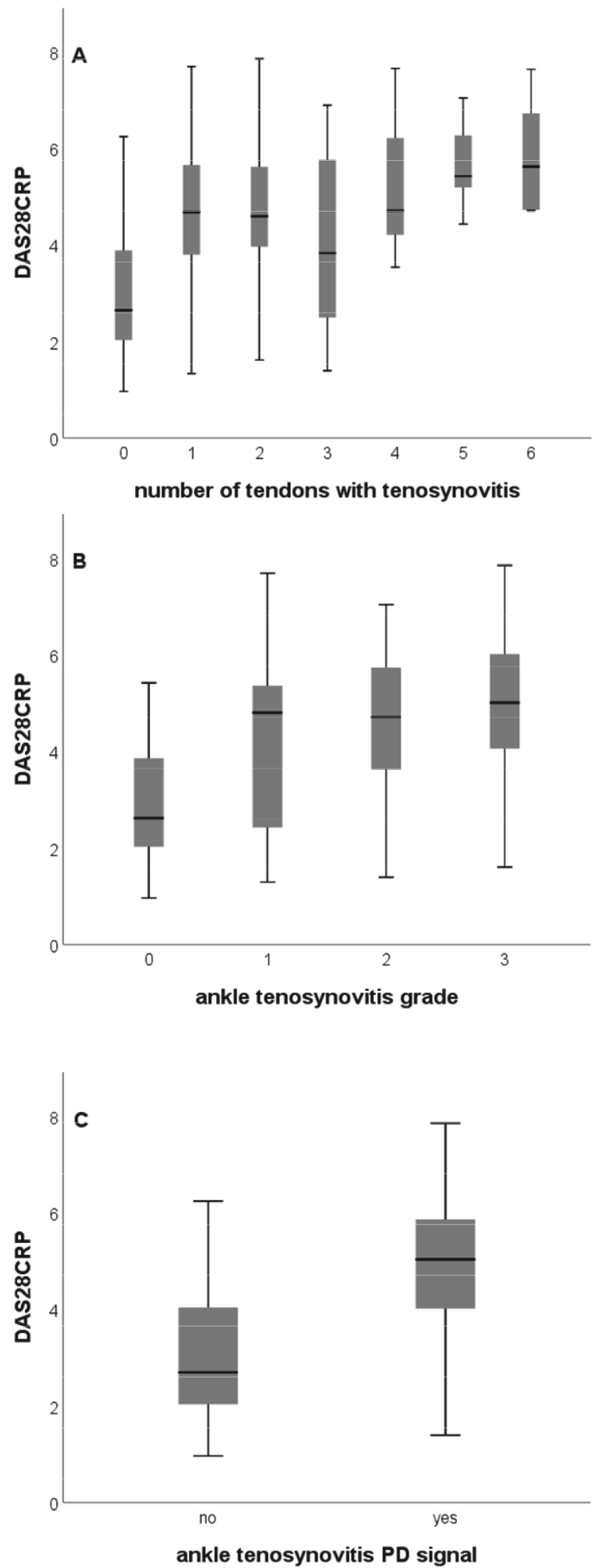

A. Categories contain the number of patients with 0 to 6 different ankle tendons with TS (either left or right): " 0 " $=87$ patients with no ankle TS (median DAS28 ${ }_{\mathrm{CRP}}=2.64$ ); " $1 "=34$ patients with TS in 1 ankle tendon (for example the right $\mathrm{PB}$; median DAS28 $\left.{ }_{\mathrm{CRP}}=4.67\right) ; " 2 "=24$ (4.59); "3"=18 (3.82); "4"=7 (4.70); "5"=7 (5.41); "6" 6 6 (5.61). Kruskal Wallis test $(n=183$; statistic $=52.3,6$ degrees of freedom; $\mathrm{p}<0.001$ ) with post-hoc analysis (Bonferoni) showing that " 0 " differed significantly from " 1 " $(p=0.001), \quad$ " $2 " \quad(p=0.001), \quad$ "4" $(p=0.024), \quad " 5 " \quad(p=0.001)$ and " $6 "$ $(\mathrm{p}=0.002)$.

B. Initially, each left and right ankle tendon was graded individually. Then left and right homologous tendons were compared and the highest grade was retained for each tendon type. Finally, the highest grade of TS among all tendon types defined the patient's general ankle TS grade. There were 86 $(47.0 \%)$ grade 0 patients (median DAS28 $\left.{ }_{\mathrm{CRP}}=2.61\right), 26(14.2 \%)$ grade 1 (4.79), $32(17.5 \%)$ grade $2(4.70)$ and $39(21.3 \%)$ grade $3(5.00)$. Kruskal Wallis test $(\mathrm{n}=183$; statistic $=50.7,3$ degrees of freedom; $p<0.001$ ) with post-hoc analysis (Bonferoni) showing that " 0 " differed significantly from " 1 " $(p=0.001), \quad " 2 " \quad(p<0.001)$ and " 3 " $(\mathrm{p}=0.001)$.

C. There were $78(42.6 \%)$ patients without PD signals in all ankle tendons (median DAS28 $8_{\mathrm{CRP}}=2.69$ ) and 105 (57.4\%) patients with PD signals in at least one ankle tendon (DAS28 $\mathrm{CRP}=$ 5.02; $\mathrm{p}<0.001 ;$ Mann Whitney test).

FIGURE 1. Median DAS28CRP according to the number of tendons with TS (A), grade of ankle TS (B) and presence of $P D$ signal in ankle TS (C).

CRP - C-reactive protein; DAS - disease activity score; PB - peroneus brevis; PD - power Doppler; TS - tenosyvitis

tokines: Takeuchi et al. [22] showed that RF titres correlate with tumour necrosis factor levels. This will infer that TP tenosynovitis, just like RF, is an expression of RA pathogenesis and might share the same specificity and prognostic ability, assumptions which merit further study. 
Wakefield et al. [23] compared clinical examination and ultrasound with high field magnetic resonance imaging (MRI) and reported that, compared to the gold standard of MRI, clinical examination was sensitive but ultrasound more specific in identifying hindfoot tenosynovitis. However, we observed low sensitivity of clinically tender and swollen ankles to reveal ultrasound-defined tenosynovitis and high specificity of swollen ankles, which are a more powerful predictor of tenosynovitis. Because of complicated anatomy and multiple confounding conditions, it seems that clinical ankle evaluation should be complemented by ultrasound scans in order to avoid false negative and false positive results. For a trained rheumatologist, ankle ultrasound should not complicate the clinical management, especially in the era of high performance machines and standardized recommendations.

Regarding the association of ankle tenosynovitis with disease activity, we observed further proof of causality: on one hand, not only the presence of tenosynovitis, but also its grade and the extent of its ankle involvement were associated with higher disease activity; on the other hand, the absence of ankle tenosynovitis was significantly and independently associated with lower disease activity measured by DAS28 ${ }_{\text {CRP }}$ The fact that Gutierrez et al. [7] did not observe this evidence could be due to its absence from their study sample, which was more tightly controlled regarding disease activity: compared to our sample, their patients had a lower mean disease duration (3.3 versus 11.2 years) and DAS28 (3.6 versus 4.4, assuming that the authors used ESR to calculate this score), a higher prevalence of csDMARD (95.4\% versus $84.7 \%)$ and glucocorticoid $(27.7 \%$ versus $17.5 \%$ ) treatment. This highlights the impor-

\section{REFERENCES}

1. Prevoo ML, van , $t$ Hof MA, Kuper $H H$, van Leeuwen MA, van de Putte LB, van Riel PL. Modified disease activity scores that include twenty-eight-joint counts. Development and validation in a prospective longitudinal study of patients with rheumatoid arthritis. Arthritis Rheum. 1995;38(1):44-8.

2. Yano K, Ikari K, Inoue E, Sakuma Y, Mochizuki T, Koenuma N et al. Features of patients with rheumatoid arthritis whose debut joint is a foot or ankle joint: A 5,479-case study from the IORRA cohort. PLoS One. 2018;13(9):e0202427.

3. van Tuyl LH, Britsemmer K, Wells GA, Smolen JS, Zhang B, Funovits $\mathrm{J}$ et al. Remission in early rheumatoid arthritis defined by 28 joint counts: limited consequences of residual disease activity in the forefeet on outcome. Ann Rheum Dis. 2012;71(1):33-7.

4. Toyota Y, Tamura M, Kirino Y, Sugiyama Y, Tsuchida N, Kunishita Y et al. Musculoskeletal ultrasonography delineates ankle symptoms in rheumatoid arthritis. Mod Rheumatol. 2017; $27(3): 425-9$ tance of study population characteristics (Table 1) and their variability: our 183 patients were predominantly women in their $6^{\text {th }}$ decade, with established RA judging by mean disease duration and relatively low frequency of seropositivity. Only to similar populations of RA patients can our results be extrapolated without increasing the risk of improper generalization. Further studies on the subject of clinical and ultrasound ankle involvement could focus on specific RA populations, such as early RA or treatment-naïve RA, in order to complement and ultimately confirm our observations.

Several study limitations should be taken into consideration when assessing the relevance of our results: the cross sectional study design, the lack of an inter-observer study, the fact that clinical examination was not done individually at the level of each ankle structure (joint, tendon) and the fact that bone damage (erosions) was not evaluated either by conventional radiography or ultrasound.

\section{CONCLUSIONS}

The most frequent ankle tenosynovitis observed in RA patients involves the TP tendon, which is associated with higher titres of RF but not ACPA. Comparing the ability of ankle tenderness and swelling to detect ultrasound-defined ankle tenosynovitis showed that swollen ankles are more specific and better predictors. Ultrasound-detected ankle tenosynovitis has a directly proportional relationship with disease activity in RA. A new DAS is needed, in order to reflect the clinical reality of today's RA patients, a DAS which should include clinical evaluation of ankles and ultrasound information.

Conflict of interest: none declared Financial support: none declared

5. Hennessy K, Woodburn J, Steultjens M. Clinical practice guidelines for the foot and ankle in rheumatoid arthritis: a critical appraisal. J Foot Ankle Res. 2016;9:31.

6. Elsaman AM, Mostafa ES, Radwan AR. Ankle Evaluation in Active Rheumatoid Arthritis by Ultrasound: A Cross-Sectional Study. Ultrasound Med Biol. 2017;43(12):2806-13.

7. Gutierrez M, Pineda C, Salaffi F, Raffeiner B, Cazenave T, Martinez-Nava GA et al. Is ankle involvement underestimated in rheumatoid arthritis? Results of a multicenter ultrasound study. Clin Rheumatol. 2016;35(11):2669-78.

8. Suzuki T, Tohda E, Ishihara K. Power Doppler ultrasonography of symptomatic rheumatoid arthritis ankles revealed a positive association between tenosynovitis and rheumatoid factor. Mod Rheumatol. 2009;19(3):235-44.

9. Suzuki T, Okamoto A. Ultrasound examination of symptomatic ankles in shorter-duration rheumatoid arthritis patients often reveals tenosynovitis. Clin Exp Rheumatol. 2013;31(2):281-4. 
10. Barn R, Turner DE, Rafferty D, Sturrock RD, Woodburn J. Tibialis posterior tenosynovitis and associated pes plano valgus in rheumatoid arthritis: electromyography, multisegment foot kinematics, and ultrasound features. Arthritis Care Res (Hoboken). 2013;65(4):495-502.

11. Hammer HB, Kvien TK. Ultrasonography shows significant improvement in wrist and ankle tenosynovitis in rheumatoid arthritis patients treated with adalimumab. Scand J Rheumatol. 2011;40(3):178-82.

12. Enache L, Popescu CC, Codreanu C, Suta M. Clinical Ankle Involvement and Ultrasound Synovial Hypertrophy are Significant Predictors of DAS28-Defined Rheumatoid Arthritis Disease Activity. Internal Medicine. 2019;16(2):19-33.

13. Aletaha D, Neogi T, Silman AJ, Funovits J, Felson DT, Bingham CO, 3rd et al. 2010 rheumatoid arthritis classification criteria: an American College of Rheumatology/European League Against Rheumatism collaborative initiative. Ann Rheum Dis. 2010;69(9):1580-8.

14. Orr CK, Najm A, Young F, McGarry T, Biniecka M, Fearon U et al. The Utility and Limitations of CRP, ESR and DAS28-CRP in Appraising Disease Activity in Rheumatoid Arthritis. Front Med (Lausanne). 2018;5:185.

15. Smolen JS, Breedveld FC, Schiff MH, Kalden JR, Emery P, Eberl G et al. A simplified disease activity index for rheumatoid arthritis for use in clinical practice. Rheumatology (Oxford). 2003;42(2):244-57.

16. Naredo E, D'Agostino MA, Wakefield RJ, Moller I, Balint PV, Filippucci $E$ et al. Reliability of a consensus-based ultrasound score for tenosynovitis in rheumatoid arthritis. Ann Rheum Dis. 2013;72(8):1328-34.

17. Barn R, Brandon M, Rafferty D, Sturrock RD, Steultjens M, Turner DE et al. Kinematic, kinetic and electromyographic response to customized foot orthoses in patients with tibialis posterior tenosynovitis, pes plano valgus and rheumatoid arthritis. Rheumatology (Oxford). 2014;53(1):123-30.

18. Simonsen MB, Yurtsever A, Naesborg-Andersen K, Leutscher PDC, Horslev-Petersen K, Hirata RP et al. A parametric study of effect of experimental tibialis posterior muscle pain on joint loading and muscle forces-Implications for patients with rheumatoid arthritis? Gait Posture. 2019;72:102-8.

19. Popelka S, Hromadka R, Vavrik P, Stursa P, Pokorny D, Jahoda D et al. Isolated talonavicular arthrodesis in patients with rheumatoid arthritis of the foot and tibialis posterior tendon dysfunction. BMC Musculoskelet Disord. 2010;11:38.

20. Bas S, Genevay S, Meyer O, Gabay C. Anti-cyclic citrullinated peptide antibodies, IgM and IgA rheumatoid factors in the diagnosis and prognosis of rheumatoid arthritis. Rheumatology (Oxford). 2003;42(5):677-80.

21. Humphreys JH, van Nies JA, Chipping J, Marshall T, van der Helm-van Mil AH, Symmons DP et al. Rheumatoid factor and anticitrullinated protein antibody positivity, but not level, are associated with increased mortality in patients with rheumatoid arthritis: results from two large independent cohorts. Arthritis Res Ther. 2014;16(6):483.

22. Takeuchi T, Miyasaka N, Inui T, Yano T, Yoshinari T, Abe T et al. High titers of both rheumatoid factor and anti-CCP antibodies at baseline in patients with rheumatoid arthritis are associated with increased circulating baseline TNF level, low drug levels, and reduced clinical responses: a post hoc analysis of the RISING study. Arthritis Res Ther. 2017;19(1):194.

23. Wakefield RJ, Freeston JE, O'Connor P, Reay N, Budgen A, Hensor EM et al. The optimal assessment of the rheumatoid arthritis hindfoot: a comparative study of clinical examination, ultrasound and high field MRI. Ann Rheum Dis. 2008; 67(12):1678-82. 